\title{
Das Leipziger Erwägungsseminar. Ein didaktisch reflektierender Erfahrungsbericht
}

\section{Lara Boerger}

\section{Zusammenfassung}

Von 2006 bis 2019 wurde an der Universität Leipzig das sog. Erwägungsseminar angeboten. Das Konzept des Seminares fußt auf der Erwägungsdidaktik der „Forschungsgruppe Erwägungskultur Paderborn“ und wurde Ende der 1980er Jahre entwickelt. Die Grundidee umreißt einen spezifischen erwägungsorientierten Umgang mit Alternativen. Demnach soll das Wissen von Expertinnen und Experten nicht als gesetzt, gesichert, und unumstößlich gelten. Vielmehr geht es darum, Theorien $\mathrm{zu}$ hinterfragen, $\mathrm{zu}$ vergleichen und einen integrierten und bewahrenden Umgang mit unterschiedlichen Erklärungsansätzen zu praktizieren. Die zentralen Forderungen des Netzwerk Plurale Ökonomik - Selbstkritik, Reflexion und Offenheit in der VWL zu fördern - werden aufgegriffen und stellen meines Erachtens das vorrangige Ziel der Lehrveranstaltung dar.

\section{Schlüsselbegriffe}

Erwägungsseminar · Erwägungsdidaktik · Plurale Ökonomik

L. Boerger $(\bowtie)$

Hochschule für Wirtschaft und Gesellschaft Ludwigshafen, Heidelberg, Deutschland E-Mail: Lara.Boerger@hwg-lu.de 


\section{$1 \quad$ Einleitung}

Während meines Masterstudiums der VWL an der Universität Leipzig habe ich das sogenannte ,Erwägungsseminar" besuchen dürfen. Als Kritikerin der aktuellen ökonomischen Hochschullehre und als langjährige Unterstützerin der Bewegung der Pluralen Ökonomik war ich positiv überrascht von diesem Lehrkonzept. Hier fand ich ein Seminar vor, welches eigenes Denken und aktive Mitgestaltung auf eine Weise fordert, wie es in der gängigen ökonomischen Hochschulbildung selten vorzufinden ist.

Ich habe das Seminar im Rahmen meines Studiums gleich zweimal besucht ${ }^{1}$. Im ersten Seminar lasen wir gemeinsam Pikettys „Kapital“ (Piketty 2014) und diskutierten seine Theorie der kapitalistischen Eigendynamik von Ungleichheit. Im nachfolgenden Semester behandelten wir einen Diskussionsbeitrag von Riese (2000) aus der Erwägungszeitschrift „Erwägen Wissen Ethik“, welcher sich mit dem Thema Geld als ,unverstandene Kategorie der Nationalökonomie“ beschäftigte. Im Folgenden möchte ich - basierend auf diesen Erfahrungen - die Entstehung und die theoretischen Grundlagen des Seminars darstellen und die methodische Ausgestaltung hinsichtlich ihres Innovationspotentials für die Lehre in der Pluralen Ökonomik reflektieren.

\section{Entstehungskontext}

Das Leipziger Erwägungsseminar fußt auf der Erwägungsdidaktik der Forschungsgruppe Erwägungskultur Paderborn. Das Seminar wurde von Friedrun Quaas und Georg Quaas im Rahmen der wirtschaftswissenschaftlichen Lehre an der Universität Leipzig seit dem Sommersemester 2006 durchgeführt und kontinuierlich weiterentwickelt. Mit einer einzigen Ausnahme fand das Seminar in jedem Semester statt, wird jedoch aufgrund fehlender Nachfolge im Jahr 2019 eingestellt.

Die Grundidee umreißt einen spezifischen erwägungsorientierten Umgang mit Alternativen und wurde Ende der 1980er Jahre von Frank Benseler, Bettina Blanck, Rainer Greshoff und Werner Loh entwickelt. Die Gruppe verfolgte

\footnotetext{
${ }^{1}$ Dies war möglich, da sowohl das Modul „Evolutorische Ökonomik“ von Friedrun Quaas als auch das Modul „Internationale Politik und Wirtschaft“ von Georg Quaas den Besuch des Erwägungsseminars mit anschließender Hausarbeit als Seminarleistung anerkannten.
} 
zunächst das Ziel, das Erwägungskonzept als Forschungstradition zu etablieren. Gemeinsam veröffentlichten sie die Diskussionszeitschrift Erwägen Wissen Ethik (EWE), in welcher der Erwägungsgedanke als paradigmatische Grundlage herangezogen wurde (Blanck 2002, S. 333). ${ }^{2}$ In diesem Rahmen entstand die Idee, den Ansatz nicht nur in der Forschung, sondern auch in der Lehre zu nutzen. Konkret geht es bei dem Konzept darum, den Studierenden ,einen entsprechenden Raum für das Denken in Alternativen“ (Ferger et al. 2008, S. 6) zu eröffnen. Dabei soll das Wissen von Expertinnen und Experten nicht als gesetzt, gesichert und unumstößlich gelten. Vielmehr geht es darum, Theorien zu hinterfragen, zu vergleichen und einen ,integrierten und bewahrenden“ Umgang mit Alternativen zu praktizieren (Blanck 2002, S. 11 f.). „Der Kerngedanke des Erwägungskonzepts besteht darin, dass sich die in einem Entscheidungszusammenhang erwogenen und dann bewahrten Alternativen als eine Geltungsbedingung für die schließlich gesetzte Lösung [...] nutzen lassen“ (ebd., S. 9).

Demnach sollen kontroverse Alternativen nicht nur vermehrt behandelt werden. Vielmehr sollen Entscheidungswissen und Entscheidungskompetenzen gestärkt und die Teilnehmenden befähigt werden, jeweilige Lösungsvorschläge bzw. Positionen mit Blick auf die Erwägungsgeltungsbedingungen einzuschätzen. Diese Vorgehensweise grenzt sich damit klar von etablierten Forschungstraditionen eines eher „kämpfenden“ Umgangs mit Alternativen ab, in welcher ein einziger Lösungsansatz gefunden und bewahrt werden soll (ebd., S. 12). Da Alternativen als Geltungsbedingung betrachtet werden, erhalten diese eine bleibende Funktion auch über die Setzung einer „besten Lösung“ hinaus zugesprochen.

An dieser Stelle verweist Blanck (2002, S. 18) auf das Wissenschaftsverständnis von Niklas Luhmann. Für diesen hängt die „Qualität“ einer Entscheidung nicht nur von der ,gewählten Alternative“ ab. „Die Identität eines Entscheidungsaktes profiliert sich deshalb nicht nur in der gewählten Alternative, sondern auch gegen den Horizont anderer Möglichkeiten, vor denen sie bevorzugt worden ist“ (Luhmann 1981, S. 338). Auch für Paul Feyerabend tragen widerlegte Theorien „zum Gehalt ihrer siegreichen Konkurrenten bei“ (Feyerabend 1981, S. 130). Blanck (2002) betont, dass eher jene Forscherinnen und Forscher, welche einem relativistischen Pluralismus zuneigen, erwogenen Alternativen im Forschungsprozess eine bleibende Funktion zusprechen. Anhängerinnen und Anhänger eines

\footnotetext{
${ }^{2}$ Von 1990 bis 2001 erschien die Zeitschrift unter dem Titel Ethik und Sozialwissenschaften (EuS).
} 
tendenziell geradlinigen Fortschrittsverständnisses hingegen zeigen laut Blanck (2002) weniger Bestreben, erwogene Alternativen zu bewahren.

„In Erwägungsseminaren sollen Konzepte unter expliziter Berücksichtigung von jeweiligen Alternativen erarbeitet werden. Die Studierenden sollen lernen, alternative Lösungen als solche zu erkennen, zu bestimmen und vergleichend mit ihnen umzugehen. Sie sollen weiterhin befähigt werden, sich eigene Positionen im Wissen um jeweilige Alternativen zu erarbeiten und in Diskussionen erwägungsoffen und korrekturbereit zu argumentieren“ (Blanck 1998, S. 169).

Das Konzept des Erwägungsseminars wurde in verschiedenen Fachbereichen erprobt. Neben dem Leipziger Seminar in Wirtschaftswissenschaften wurden an anderen Hochschulen Veranstaltungen in Erziehungswissenschaften, Informatik, Soziologie und katholischer Theologie durchgeführt. ${ }^{3}$ Die Ausgestaltung kann im Einzelnen sehr unterschiedlich sein. Beispielsweise gab es stark literaturorientierte Seminare ebenso wie solche, die keine verbindliche Literatur erarbeiteten. Auch können Erwägungsseminare stark studierendenzentriert gestaltet sein oder eher lehrerzentriert arbeiten (Blanck 2002, S. 301-304). Der folgende Beitrag stellt die Erfahrungen aus dem Leipziger Erwägungsseminar dar. Er zeigt, dass die Herangehensweise der Erwägungsdidaktik besonders im Bereich der Wirtschaftswissenschaften eine Bereicherung darstellen kann. Die zentralen Forderungen des Netzwerk Plurale Ökonomik - Selbstkritik, Reflexion und Offenheit in der VWL zu fördern ${ }^{4}$ - werden aufgegriffen und stellen meines Erachtens das vorrangige Ziel der Lehrveranstaltung dar. Auch Quaas und Quaas selbst betonen die Bedeutung für eine pluralere Hochschullehre, welche der Theorienvielfalt der Ökonomik gerecht werden soll: „Angesichts der Theorienpluralität in den Wirtschaftswissenschaften und des Vorherrschens eines Mainstreams, der sich durch kaum etwas anderes als durch wissenschaftsinstitutionelle Machtpositionen legitimiert, wäre ein stärkeres Vordringen hin zu erwägenden Lehrveranstaltungen eine Bereicherung" (Quaas und Quaas 2012, S. 3).

\footnotetext{
${ }^{3}$ Beispiele und Berichte finden sich in der Diskussionszeitschrift Erwägen Wissen Ethik oder online: https://groups.uni-paderborn.de/ewe/ [29.08.2019].

${ }^{4}$ Nähere Informationen zum Netzwerk Plurale Ökonomik online: https://www.pluraleoekonomik.de/ [29.08.2019].
} 


\section{Lehrinnovation}

Das Leipziger Erwägungsseminar fand, wie bereits erwähnt, über einen Zeitraum von zehn Jahren an der Universität Leipzig statt und wurde in diesem Rahmen kontinuierlich weiterentwickelt und ausführlich dokumentiert. Auf der seminareigenen Internetseite ${ }^{5}$ finden sich sowohl Protokolle der Sitzungen als auch Erfahrungsberichte, die das Seminar selbst evaluieren und reflektieren. In den letzten Jahren hat sich ein fester Rahmen etabliert, welcher das Leipziger Erwägungsseminar maßgeblich ausmacht:

- Alle Teilnehmenden sind gleichberechtigt. Die Dozierenden nehmen keine richtungsweisende Position ein.

- Um eine gleichberechtigte Gesprächskultur zu fördern, wird eine kreisförmige Sitzordnung hergestellt.

- $\mathrm{Zu}$ jeder Sitzung wird zu Beginn eine Tagesordnung vorgeschlagen und gemeinsam beschlossen.

- Der konkrete Umfang und Inhalt der Lektüre für die nächste Sitzung werden am Ende einer Sitzung festgelegt.

- Die inhaltliche Sitzung beginnt mit einem Statement eines Teilnehmenden zum gelesenen Text. Wer das Statement hält, entscheidet das Los und kann damit auch die Dozierenden treffen.

- Das Seminar wird protokolliert und das Protokoll auf der seminareigenen Internetseite veröffentlicht.

- Wer sprechen will, ergreift das Wort.

- Alle verpflichten sich, regelmäßig am Seminar teilzunehmen.

Dieses Regelsystem wurde immer zu Beginn der Veranstaltungsreihe vorgestellt und ebenfalls gemeinsam beschlossen. Natürlich konnten alle Teilnehmenden Änderungen einbringen. Beispielsweise war es möglich, eine Moderation einzuführen und Redelisten zu nutzen, worauf erfahrungsgemäß aber tendenziell verzichtet wurde. Auch im Laufe des Seminars konnten jederzeit Verfahrensvorschläge eingebracht werden.

Die ,herrschaftsfreie Kommunikation" stellte eine der wesentlichen Erkennungsmerkmale des Leipziger Erwägungsseminares dar. Demnach wurden die ,sonst herrschenden Riten der Worterteilung und der autoritativen Lenkung der Diskussion außer Kraft gesetzt“, nachdem in der ersten Sitzung die Rahmen-

${ }^{5}$ Siehe online: http://evoeco.forschungsseminar.de/ [29.08.2019]. 
bedingungen erläutert und organisatorische Fragen geklärt wurden (Ferger et al. 2008, S. 6). Diese nicht-autoritäre Struktur sollte jedoch nicht mit Regellosigkeit verwechselt werden. Vielmehr kann von einer basisdemokratischen Organisationsstruktur gesprochen werden. Die anfängliche Themenwahl und die Beurteilung der Seminarleistung in Form einer Hausarbeit ist davon jedoch ausgenommen und wird - wie üblich - von den Dozierenden vorgenommen. Diese Ausnahmen sind notwendig, um eine Einbettung in das ,expertokratisch organisierte Universitätssystem“ (ebd., S. 6) zu ermöglichen.

Thematisch waren die Seminare in das Lehrmodul „Ökonomische und politische Bedingungen der Globalisierung“ integriert. Unter diesem eher allgemeinen Rahmenthema wurden jedes Semester neue Themeninhalte behandelt, mit dem Ziel das Phänomen der Globalisierung aus verschiedenen Perspektiven zu erörtern. Anfangs orientierten sich die Erwägungsinhalte an den Diskussionseinheiten der Zeitschrift Erwägen Wissen Ethik (EWE). ${ }^{6}$ Diese erwiesen sich als besonders geeignet, da die Texte dem von EWE eigens entwickelten „ThesenKritik-Replik"-Verfahren folgten und die Erwägungsstruktur gewissermaßen vorgegeben war. ${ }^{7}$ Später wurden auch externe Inhalte behandelt, bei denen Monografien oder thematisch zusammenhängende Texte den Ausgangspunkt für den Erwägungsprozess bildeten (Quaas und Quaas 2012, S. 1). ${ }^{8}$

Die Förderung der Fachkompetenz ist Teil des didaktischen Ziels, steht jedoch nicht im Vordergrund. Neben den didaktischen Zielen, die Bettina Blanck für das Erwägungsseminar formuliert hat ${ }^{9}$, betonen Quaas und Quaas (2012, S. 2), dass vor allem die Fähigkeit zur Äußerung von Kritik gefördert werden soll. Gerade hier sehen sie in der wirtschaftswissenschaftlichen Lehrpraxis eine wesentliche Leerstelle, was zur Folge hat, dass die Vorzugswürdigkeit einer gewählten Alternative ,häufig nicht oder zumindest nicht gut argumentativ begründet“ (ebd.)

\footnotetext{
${ }^{6}$ Konkret wurden folgende Diskussionseinheiten behandelt: 1. Altvater (2002), 2. Radnitzky (2002), 3. Witt (2004), 4. Ulrich (2000) und 5. Riese (2000).

${ }^{7}$ Das Verfahren wird im späteren noch ausführlicher erläutert.

${ }^{8}$ Unter den Monografien fanden sich beispielsweise Piketty (2014), Krugman (1999) und Rodrik (2010). Darüber hinaus wurden die Themen Krisentheorien, das bedingungslose Grundeinkommen oder die Euro-Krise behandelt. Alle Erwägungsinitiale finden sich online: http://evoeco.forschungsseminar.de/ [29.08.2019].

${ }^{9}$ Diese wurden oben bereits ausführlicher dargelegt. „Die Studierenden sollen lernen, alternative Lösungen als solche zu erkennen, zu bestimmen und vergleichend mit ihnen umzugehen. Sie sollen weiterhin befähigt werden, sich eigene Positionen im Wissen um jeweilige Alternativen zu erarbeiten und in Diskussionen erwägungsoffen und korrekturbereit zu argumentieren“" (Blanck 1998, S. 169).
} 
wird. Die Seminare benötigten in der Regel keine besondere Ausstattung, jedoch sind einige Hilfsmittel von Vorteil. Besonders die zugeschnittene eigene Website ist im Laufe der Zeit zu einem ,unverzichtbaren Arbeitsinstrument“ geworden (Ferger et al. 2008, S. 5). Sie diente den Teilnehmenden als Orientierung, half bei der Koordination des Erwägungsprozesses und hatte darüber hinaus auch eine archivierende Funktion. Auch haben sich einige Methoden als hilfreich erwiesen, um den Erwägungsprozess zu strukturieren. Besondere Anwendung fand das bereits erwähnte Thesen-Kritik-Replik-Verfahren, welchem auch die Beiträge der Zeitschrift Ethik Wissen Erwägen folgten. Diese Methode dient der Strukturierung von Diskussionseinheiten, indem eine schriftliche diskursive Auseinandersetzung nach dem Schema These - Kritik - Replik durchgeführt wird. Formal bedeutet dies, dass zunächst zu einem bestimmten Thema oder einer Frage ein Thesenpapier verfasst wird. Dies kann in Einzelarbeit, Zweier-Teams oder Kleingruppen erarbeitet werden. Im zweiten Schritt tauschen diese das Thesenpapier mit anderen Seminarteilnehmerinnen und -teilnehmern und verfassen zu dem nun erhaltenen Papier eine Kritik. Die Kritiken wiederum werden den jeweiligen Autorinnen und Autoren der Thesenpapiere gegeben, welche darauf aufbauend eine Replik verfassen. ,Aus erwägungsorientierter Perspektive kommt es darauf an, dass die Teilnehmenden in ihren Beiträgen ihre jeweils vertretenen Positionen gegenüber zu erwägenden Alternativen zu begründen versuchen und das jeweilige Begründungsniveau reflektieren“ (Blanck 2006).

Auch andere erwägungsorientierte Methoden wie Begriffslisten, Erwägungstafeln oder Pyramidendiskussionen wurden im Rahmen des Seminars ausprobiert. ${ }^{10}$ Während einige Methoden zum festen Bestandteil des Seminars wurden und immer wieder Anwendungen fanden, wurden andere als weniger hilfreich empfunden. So scheiterten die Pyramidendiskussionen beispielsweise daran, dass mögliche Alternativen nicht ausreichend inhaltlich identifiziert wurden und die Ausarbeitung einer Pyramide bereits an der Basis Probleme bereitete. „Waren wir vor Jahren noch zuversichtlich angetreten, möglichst viele Erwägungsmethoden testen zu können, zeigte sich letztlich doch rasch, dass die Grenzziehung viel früher einsetzte. Die von Bettina Blanck empfohlenen

\footnotetext{
${ }^{10}$ Einige Methoden greifen auf das Konzept von Bettina Blanck zurück; einige sind im Seminar selbst entstanden. Begriffslisten beispielsweise dienen dem Zweck, Begriffe in ihrem jeweiligen Kontext auffindbar und auf diese Weise in ihrer Spezifik leichter erkennbar zu machen (Ferger et al. 2008, S. 11). Genauere Beschreibungen der Methoden und weitere Vorschläge zur Erschließung vielfältiger Perspektiven finden sich auf der Internetseite des Erwägungsseminares oder bei Blanck 2002, S. 299-324.
} 
Verfahren erwägungsorientierten Studierens kamen nicht zur Gänze zum Einsatz“ (Quaas und Quaas 2015, S. 7).

Generell war zu beobachten, dass Studierende der Wirtschaftswissenschaften diesen Instrumentarien aufgeschlossener gegenüberstanden als Studierende anderer sozialwissenschaftlicher Disziplinen (Ferger et al. 2008, S. 6). Darüber hinaus fällt auf, dass das Erwägungsseminar vor allem von Studierenden der Wirtschaftswissenschaften als etwas Besonderes wahrgenommen wurde. Dies liegt darin begründet, dass sich das Seminar sehr stark von der sonst üblichen didaktischen Herangehensweise in der ökonomischen Hochschullehre unterscheidet. Als Besonderheiten wurden die demokratische Struktur, das notwendige Engagement der Einzelnen und die speziellen Regeln genannt. Diese Einschätzung teilen jedoch nicht alle. Ein Studierender der Soziologie schreibt nach dem Seminar, dass für ihn offen bleibt, durch welche Besonderheiten sich das Erwägungskonzept auszeichnet. „Insbesondere im Kontext meiner bisherigen Seminarerfahrungen in der Soziologie stellt sich mir die Frage, warum man nicht einfach von einer sehr ausführlichen Literaturbesprechung spricht" (Ferger et al. 2008, S. 20).

\title{
$4 \quad$ Wirkungen und Folgen
}

Die ausführliche Dokumentation und Reflexion des Erwägungsseminares erlaubt es mir, nicht nur auf die eigenen Erfahrungen zurückgreifen zu müssen. Erfahrungsberichte $^{11}$ von Seiten der Dozierenden als auch von Seiten der Studierenden ermöglichen eine breitere Evaluation.

Besondere Aufmerksamkeit erhält in allen Berichten der Kommunikationsstil des Erwägungsseminares, welcher von Seiten der Studierenden sehr unterschiedlich bewertet wird. In Evaluationen kritisieren einige die fehlende Zielführung und ausufernde Diskussionen und sprechen sich stark für eine stärkere Strukturierung und klarere Gesprächsführung aus.

\begin{abstract}
„Als Mensch, dem die Methode des Erwägungsseminars bisher unbekannt war, sind mir vor allem die langen, oft ergebnislos erscheinenden Diskussionen aufgefallen. Allzu oft hätte ich gerne die Diskussion abgebrochen, um endlich , auf den Punkt zu kommen“ und zielführender vorzugehen." (Ferger et al. 2008, S. 20).
\end{abstract}

\footnotetext{
${ }^{11} \mathrm{Zu}$ finden unter „Publikationen“ auf der Internetseite des Erwägungsseminares (Online: http://evoeco.forschungsseminar.de/ [29.08.2019]).
} 
„Manchmal war es auch so, dass alles so lange zerpflückt wurde, bis man völlig den Faden verloren hat und sich fragen musste, worum es gleich noch mal ging, was der Grund für diese Diskussion war" (ebd.).

Auf der anderen Seite ist es gerade die gleichberechtigte Diskussionskultur, welche von anderen Studierenden besonders positiv bewertet wird.

„Innerhalb des Seminars konnten wir uns in einer Diskussionskultur üben, welche nicht ständig von einem Pol dominiert wird“ (ebd.).

„Meiner Erfahrung nach trauen sich zu viele Studierende auch am Ende ihres Studiums nicht, einen Prof. Dr. Dr. zu kritisieren, und gehen davon aus, dass dieser aufgrund seines Titels die endgültige Wahrheit verkündet“" (ebd.).

Auch ich selbst erlebte es als bereichernd, dass in der Diskussion keine konkreten Antworten erwartet wurden und der Prozess offen von den Teilnehmenden bestimmt wurde. In anderen Seminaren hatte ich oft den Eindruck, dass die Dozierenden bestimmte Argumente erwarteten und der Diskussion damit im Vorhinein schon die Tiefe genommen wurde. Im Erwägungsseminar wurde ich zum ersten Mal im Rahmen einer universitären Veranstaltung aufgefordert, selbstständig zu denken und die Gedanken zu bestehenden Theorien eigenständig zu formulieren.

Gleichwohl fällt auf, dass im Erwägungsseminar häufig eine Sprachkultur vorherrschte, bei welcher vor allem Redegewandtheit und rhetorische Dominanz obsiegten. Zwar bestand jederzeit die Möglichkeit eine Moderation einzuführen, jedoch zeigte die Erfahrung, dass nur selten hierauf zurückgegriffen wurde. Eine Moderation als Bestandteil des anfänglichen Regelsystems fest zu etablieren bzw. vorzuschlagen, erscheint mir daher gerade in Anbetracht dieser Beobachtung ratsam. Diese könnte gegebenenfalls eine gleichberechtigtere Beteiligung bewirken. Idealerweise wechselt die Moderation in jeder Sitzung, sodass alle Teilnehmenden diese Rolle mindestens einmal übernehmen. Die Moderation sollte Wortbeiträge von jenen bevorzugt behandeln, welche sich seltener einbringen. Gleichzeitig könnte sie ausufernde Diskussionen und wiederkehrende Argumente benennen und unterbinden.

Darüber hinaus könnte die Moderation auch eine stärkere Strukturierung vorantreiben. Gute Erfahrungen habe ich persönlich mit der Nutzung von Moderationskarten gemacht, mit welchen die Diskussion zeitgleich kartiert und somit für alle visualisiert werden kann. Dieser Methode folgend werden zentrale Begriffe, Konzepte oder Thesen auf Moderationskarten niedergeschrieben und gesammelt. Ziel ist die Reduktion komplexer Sachverhalte auf wesentliche 
Argumente und Schlüsselbegriffe. Gleichzeitig erfolgt auch eine Strukturierung, sodass die Beziehung der Karten zueinander bestimmt und für alle festgehalten wird. Die Nutzung von Moderationskarten birgt den Vorteil, dass die Anordnung leicht veränderbar ist, sodass gemeinsame Abstimmungsprozesse angeregt werden und zeitgleich eingearbeitet werden können.

Eine gemeinsame Dokumentation auf diese Weise könnte eine stärkere Strukturierung ermöglichen, ohne die Dozierenden wieder in eine lenkende Rolle zu versetzen. Gleichzeitig hilft die gemeinsame Strukturierung, von der inhaltlichen Diskussion zu abstrahieren und auf der Metaebene Verfahrensvorschläge über das weitere Vorgehen zu machen, sodass auch hier eine stärkere Beteiligung der Studierenden gefördert wird. In den Seminaren, die ich besucht habe, wurde das Protokoll - als gemeinsame Ergebnissicherung - von Georg Quaas verfasst und veröffentlicht. Die Studierenden wollten in der Regel weder die Protokollierung des Seminars übernehmen, noch wurde das Protokoll von vielen Teilnehmenden im Nachhinein gelesen. Eine gemeinsame Dokumentation könnte an dieser Stelle helfen, eine stärkere Strukturierung zu erreichen und den roten Faden für alle sichtbarer zu machen.

Dieser Vorschlag hat jedoch den Nachteil, dass der Arbeitsaufwand für die Studierenden nochmals größer wird. Dabei ist der Arbeitsaufwand ein seitens der Studierenden oftmals angemahnter Aspekt. „Damit ein Erwägungsseminar wirklich gut verläuft, braucht es mehr Vor- und Nachbereitung [...] als in normalen Vorlesungen/Seminaren" schreibt ein Teilnehmer des Erwägungsseminares (Ferger et al. 2008, S. 21). Auch ich habe beobachtet, dass das Seminar vor allem von denjenigen Teilnehmenden gestaltet wurde, welche ein besonderes Engagement und eine große Arbeitsbereitschaft an den Tag legten. Unzureichend vorbereitete Studierende blieben in der Diskussion außen vor und brachten sich immer weniger ein. Quaas und Quaas (2015, S. 6) beschreiben, dass auf Seiten der Dozierenden insbesondere die Suche nach einem geeigneten Seminarthema mit Aufwand verbunden ist, sobald externe Erwägungsinhalte abseits der Diskussionszeitschrift Erwägen Wissen Ethik eingeführt werden. Der gesamte Arbeitsaufwand lässt sich jedoch schlecht abschätzen, da der Erwägungsprozess offen ist und sich erst im Laufe des Seminars zeigt, welche zusätzlichen Vor- und Nachbereitungen notwendig werden.

Vor allem zu Beginn der Leipziger Erwägungsseminare stand das gemeinsame Forschen im Fokus der Veranstaltung. So wurden 2007 und 2008 gemeinsame Seminarberichte verfasst und in der Zeitschrift Erwägen Wissen Ethik veröffentlicht. Auch wenn in den Seminaren, welche ich besucht habe, keine gemeinsame Veröffentlichung stattfand, hatte ich dennoch den Eindruck, forschend tätig zu sein, statt lediglich an einer Lehrveranstaltung teilzunehmen. Beide Hausarbeiten, 
welche ich im Rahmen der Erwägungsseminare schrieb, enthielten einen deutlich größeren Eigenanteil als die ,Zusammenfassungen“ früherer Seminararbeiten. Dies führe ich auf die Erwägungsmethode an sich zurück, welche eine kritischere Auseinandersetzung mit den zusammengetragenen Argumenten fördert. Die vielen Seminarsitzungen und Diskussionen regten eigene Überlegungen an, welche ich am Ende für meine Hausarbeiten nutzen konnte.

Auch wenn die Erwägungszeitschrift EWE nicht mehr herausgegeben wird, sollten zukünftige Erwägungsseminare verstärkt Möglichkeiten nutzen, um erarbeitete Inhalte zu veröffentlichen. Die Aussicht auf eine Veröffentlichung könnte die Motivation der Teilnehmenden erhöhen. Gleichzeitig werden Ergebnisse auch nach dem Seminar nutzbar und für andere einsehbar gemacht. Noch immer besteht beispielsweise die Möglichkeit, Beiträge auf der Internetseite der Forschungsgruppe Paderborn unter der Rubrik Erwägungslabor zugänglich zu machen. ${ }^{12}$ Außerdem könnten Artikel der freien Enzyklopädie Wikipedia überarbeitet und eigene Beiträge an dieser Stelle veröffentlicht werden. Auch eine Zusammenarbeit mit der pluralen Online-Lernplattform Exploring Economics wäre an dieser Stelle denkbar.

Quaas und Quaas (2015, S. 7) beschreiben das Erwägungsseminar selbst im letzten Erfahrungsbericht als ambivalentes Experiment. Einige Standards, die das Konzept erfordert hätte, konnten auf Grund des institutionellen Rahmens nicht in Gänze umgesetzt werden. Zu nennen ist hier sicherlich die Verschulung der universitären Lehre durch die Einführung der Bachelor- und Masterstudiengänge. So sahen viele Studierende keinen Anreiz darin, Inhalte zu studieren, die über den Pflichtkanon hinausgehen. Darüber hinaus waren die Teilnehmenden trotz guten Willens oft nicht in der Lage, ,,sich voll und ganz auf eine kritische inhaltliche Alternativenschau einzulassen“ (ebd.). Dies hatte zur Folge, dass am Ende eines Seminars vieles ,Stückwerk' blieb, von dem man zu Beginn dachte, dass es einer Problemlösung nahekommen könnte. In der Bilanz überwogen aber die positiven Eindrücke. Das gute Feedback der Studierenden ermunterte immer wieder, „das Konzept nicht aufzugeben, am Leben zu erhalten und an gegebene Bedingungen anzupassen“ (ebd.).

Ich selbst hoffe, mit diesem Beitrag zum Erhalt und zur Verbreitung des Erwägungskonzeptes beizutragen. Auch wenn das Erwägungsseminar in Leipzig keine Nachfolge gefunden hat, könnten sich in der neuen Generation von Hochschullehrenden, die dem sozioökonomischen Pluralismus zugetan sind, einige Vertreterinnen und Vertreter finden, die eine Weiterführung dieser didaktischen

${ }^{12}$ Online: https://groups.uni-paderborn.de/ewe/ [29.08.2019]. 
Methode als pädagogisch sinnvoll und gewinnbringend betrachten. Meines Erachtens realisiert die Erwägungsdidaktik die Forderung nach mehr Pluralismus in der ökonomischen Hochschullehre derart treffend, dass die fehlende Bekanntheit bei Unterstützerinnen und Unterstützern der Pluralen Ökonomik fast schon verwundert.

\section{Literatur}

Altvater, E. (2002). Kapitalismus. Zur Bestimmung, Abgrenzung und Dynamik einer geschichtlichen Formation. Erwägen Wissen Ethik, 13, 281-292.

Blanck, B. (1998). Erwägen als philosophische Orientierung und Didaktik. In K. Reinhard \& T. Schmidt (Hrsg.), Akademische Philosophie zwischen Anspruch und Erwartung (S. 164-195). Frankfurt/M.: Suhrkamp.

Blanck, B. (2002). Erwägungsorientierung, Entscheidung und Didaktik. Erwägungskultur in Forschung, Lehre und Praxis Bd. 2. Stuttgart: Lucius \& Lucius.

Blanck, B. (2006). Didaktische Hinweise zum Thesen-Kritik-Replik-Verfahren. http:// evoeco.forschungsseminar.de/blanck065.pdf. Zugegriffen: 29. Aug. 2019.

Ferger, F., Flöth, A., Karig, M., Keller, C., Quaas, F., Quaas, G., Schenk, E., Thalemann, G., \& Thieme, S. (2008). Leipziger Erwägungsseminare - Zweiter Erfahrungsbericht zur Institutionalisierung einer alternativen Lehrform. Erwägen Wissen Ethik, 19(1), $3-24$.

Feyerabend, P. K. (1981). Probleme des Empirismus. Braunschweig/Wiesbaden: Vieweg+Teubner.

Krugman, P. (1999). Der Mythos vom globalen Wirtschaftskrieg. Frankfurt/M.: Campus.

Luhmann, N. (1981). Organisation und Entscheidung. In Ders., Soziologische Aufklärung; Bd. 3 (S. 335-389). Wiesbaden: VS Verlag für Sozialwissenschaften.

Piketty, T. (2014). Das Kapital im 21. Jahrhundert. München: C.H. Beck.

Quaas, F., \& Quaas, G. (2012). „Diskutiert wird doch in allen Seminaren...“ - Erfahrungsbericht aus den Leipziger Erwägungsseminaren. Erwägen Wissen Ethik, 23(1), 139142.

Quaas, F., \& Quaas, G. (2015). Erwägen zwischen Skylla und Charybdis - Ein (weiterer) Erfahrungsbericht aus den Leipziger Erwägungsseminaren. Erwägen Wissen Ethik, 26(1), 146-154.

Radnitzky, G. (2002). Das moralische Problem der Politik. Ethik und Sozialwissenschaften, $13,345-358$.

Riese, H. (2000). Geld - die unverstandene Kategorie der Nationalökonomie. Erwägen/ Wissen/Ethik, 7(4), 487-497.

Rodrik, D. (2010). Das Globalisierungsparadox. Die Demokratie und die Zukunft der Weltwirtschaft. München: C.H. Beck.

Ulrich, P. (2000). Integrative Wirtschaftsethik: Grundlagenreflexion der ökonomischen Vernunft. Ethik und Sozialwissenschaften, 11, 555-567.

Witt, U. (2004). Beharrung und Wandel - ist wirtschaftliche Evolution theoriefähig? Erwägen Wissen Ethik, 15, 33-45. 
Open Access Dieses Kapitel wird unter der Creative Commons Namensnennung 4.0 International Lizenz (http://creativecommons.org/licenses/by/4.0/deed.de) veröffentlicht, welche die Nutzung, Vervielfältigung, Bearbeitung, Verbreitung und Wiedergabe in jeglichem Medium und Format erlaubt, sofern Sie den/die ursprünglichen Autor(en) und die Quelle ordnungsgemäß nennen, einen Link zur Creative Commons Lizenz beifügen und angeben, ob Änderungen vorgenommen wurden.

Die in diesem Kapitel enthaltenen Bilder und sonstiges Drittmaterial unterliegen ebenfalls der genannten Creative Commons Lizenz, sofern sich aus der Abbildungslegende nichts anderes ergibt. Sofern das betreffende Material nicht unter der genannten Creative Commons Lizenz steht und die betreffende Handlung nicht nach gesetzlichen Vorschriften erlaubt ist, ist für die oben aufgeführten Weiterverwendungen des Materials die Einwilligung des jeweiligen Rechteinhabers einzuholen.

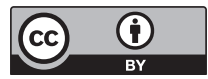

